Proceedings of the American Control Conference

San Diego, California • June 1999

\title{
A NONLINEAR MISSILE GUIDANCE CONTROLLER WITH PULSE TYPE INPUT DEVICES
}

\author{
Li-Chen Fu ${ }^{1,2}$, Chi-Wang Tsai ${ }^{1}$, Fu-Kuang Yeh $^{1}$ \\ Department of Electrical Engineering ${ }^{1}$ \\ Department of Computer Science and Information Engineering ${ }^{2}$ \\ National Taiwan University, Taipei, Taiwan, R.O.C.
}

\begin{abstract}
This paper presents a nonlinear missile guidance controller with pulse type control inputs for the interception of a theater ballistic missile in the exoatmosphere region. A 3D terminal guidance law is designed to eliminate the "sliding velocity" such that the relative motion between the missile and the target is restricted along the LOS. To enhance the performance of the missile guidance, a quaternion-based attitude controller is proposed. Sliding mode control and nonlinear system analysis theory are employed to design stable pulse type control system.
\end{abstract}

\section{Introduction}

To intercept a target in the exoatmosphere region where the density of the air is so low that the missile can not obtain sufficient maneuverability via traditional aerodynamic forces. Hence, the pulse type control inputs such as mini-dynamites or rocket engines are introduced to exert required acceleration.

The most widely used guidance algorithms are the proportional navigation guidance and its variants. Because the guidance laws required continuous control input, and can not be fulfilled with pulse type control inputs, it is necessary to design a new guidance law based on the underlying concept of the proportional navigation guidance law.

The acceleration of the missile are closely related to the attitude of the missile because the pulse type control input are fixed on the missile body. To control the attitude of the missile body with pulse type control input, a quaternion-based attitude controller is proposed.

In this paper, we present a nonlinear guidance law which is integrated with a quaternion-based attitude controller. The stability and the performance of the overall system is investigated, and the feasibility of this design is verified by simulations.

\section{System Modeling}

\section{Equations of Motion:}

The translational motion can be described via Newton's law as:

$$
\begin{array}{ll}
\dot{x}=v_{x}, & \dot{v}_{x}=\frac{1}{M} u_{x}, \\
\dot{y}=v_{y}, & \dot{v}_{y}=\frac{1}{M} u_{y}, \\
\dot{z}=v_{z}, & \dot{v}_{z}=\frac{1}{M} u_{z},
\end{array}
$$

The rotation of the missile is described by the Euler's Equation of rotation as:

$$
\dot{J} \omega+J \dot{\omega}=-\langle\omega \times\rangle J \omega+\text { Torque }
$$

where $\langle\omega \times\rangle=\left[\begin{array}{rrr}0 & -\omega_{3} & \omega_{2} \\ \omega_{3} & 0 & -\omega_{1} \\ -\omega_{2} & \omega_{1} & 0\end{array}\right]$.

\section{Quaternion:}

According to Euler's rotation theory, for two arbitrary coordinate frames $B_{1}$ and $B_{2}$ with respective origins being coincident, there exist a unit vector $p$ and an angle $\phi$ such that when $B_{1}$ undergoes an rotation of angle $\phi$ with respect to $p, B_{1}$ will coincide with $B_{2}$. Quaternion, or also named as Euler parameters, can be defined as four parameters $q=\left[\begin{array}{llll}q_{1} & q_{2} & q_{3} & q_{4}\end{array}\right]^{\top}=$ $\left[\begin{array}{ll}\bar{q}^{\top} & q_{4}\end{array}\right]^{\top}$ involving $p$ and $\phi$ :

$$
\begin{aligned}
\bar{q} & =\left[\begin{array}{l}
q_{1} \\
q_{2} \\
q_{3}
\end{array}\right]=\left[\begin{array}{l}
p_{1} \sin (\phi / 2) \\
p_{2} \sin (\phi / 2) \\
p_{3} \sin (\phi / 2)
\end{array}\right] \\
q_{4} & =\cos (\phi / 2)
\end{aligned}
$$

The relation between a rotation matrix $B_{b}$ and its corresponding quaternion $q$ follows:

$$
B_{b}=2\left[\begin{array}{ccc}
\frac{1}{2}-q_{2}^{2}-q_{3}^{2} & q_{1} q_{2}-q_{3} q_{4} & q_{1} q_{3}+q_{2} q_{4} \\
q_{1} q_{2}+q_{3} q_{4} & \frac{1}{2}-q_{1}^{2}-q_{3}^{2} & q_{2} q_{3}-q_{1} q_{4} \\
q_{1} q_{3}-q_{2} q_{4} & q_{2} q_{3}+q_{1} q_{4} & \frac{1}{2}-q_{1}^{2}-q_{2}^{2}
\end{array}\right]
$$




$$
\begin{aligned}
q_{4} & = \pm \frac{1}{2} \sqrt{B_{11}+B_{22}+B_{33}+1} \\
\bar{q} & =\mp\left[\begin{array}{c}
\frac{1}{4}\left(B_{32}-B_{23}\right) / q_{4} \\
\frac{1}{4}\left(B_{13}-B_{31}\right) / q_{4} \\
\frac{1}{4}\left(B_{21}-B_{12}\right) / q_{4}
\end{array}\right]
\end{aligned}
$$

where the sign in eq. (5) depends on the direction of the vector $\boldsymbol{p}$ and can be selected for design convenience. The error quaternion, defined as the required rotation from the initial quaternion $q_{i}$ to the desired quaternion $q_{d}$ can be described as:

$$
\begin{aligned}
\bar{q}_{e} & =\left\langle\bar{q}_{i} \times\right\rangle \bar{q}_{d}+q_{d 4} \bar{q}_{i}-q_{i 4} \bar{q}_{d} \\
q_{e 4} & =q_{i 4} q_{d 4}+\bar{q}_{i}^{\top} \bar{q}_{d}
\end{aligned}
$$

The time derivative of a quaternion is a function of the corresponding angular velocity and the quaternion itself, i.e.,

$$
\begin{aligned}
\dot{q} & =\frac{1}{2}\langle\bar{q} \times\rangle \omega+q_{4} \omega \\
\dot{q}_{4} & =-\frac{1}{2} \omega^{\top} \bar{q}
\end{aligned}
$$

Eq. (7) is a globally valid expression, and hence quaternion is more suitable in cases the controlled objects needs large-angle maneuverability.

\section{Pulse Type Input:}

In this paper, the pulse type control inputs employed are assumed to have the simple relay form as shown in Fig.(1), and the imperfection of the pulse, i.e., the delay time, the rise time, etc., are assumed to be negligible.

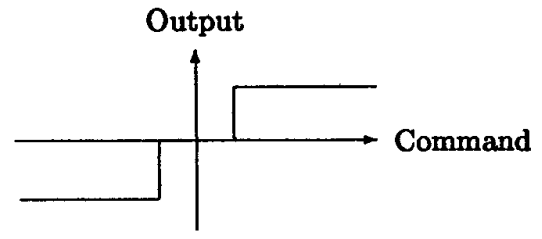

Figure 1: Pulse Type Actuator Model

It is assumed that the divert control thrusts of the missile are located near the mass center of the missile, and are aligned with the two axes perpendicular to the longitudinal axis of the kill vehicle, so as to generate the pure but arbitrary divert motion, whereas attitude control thrusts are located and aligned such that only three pure rotational moments about the the principal axes are produced. Note that those thrusts are all pulse type, i.e., they only have ON/OFF states with fixed amplitudes.

\section{Control System Design}

\subsection{Autopilot System Design}

When a missile flies in the exoatmosphere, the missile attitude will affect the control directions of DCS(Divert Control System) thrusters. Hence, in this paper, the objectives of the autopilot system include
1. to stabilize the attitude of the missile,

2. to align the DCT in the commanded directions,

3. to lock the trajectory of the target.

Given the commanded and the present attitude, define the auxiliary error $S_{a}=P \bar{q}_{e}+\omega_{e}$ where $P=$ $\operatorname{diag}\left[\begin{array}{lll}p_{1} & p_{2} & p_{3}\end{array}\right]$ is a positive definite matrix, and $\omega_{e}=\omega-\omega_{d}$ is the error angular velocity. Define a Lyapunov function candidate $V_{a}\left(S_{a}\right)$ as:

$$
V_{a}\left(S_{a}\right)=\frac{1}{2} S_{a}^{\top} S_{a}
$$

Then, the time derivative of $V_{a}\left(S_{a}\right)$ can be derived from eqs. (2) and (7) as:

$$
\begin{aligned}
\dot{V}_{a}\left(S_{a}\right) & =S_{a}^{\top} \dot{S}_{a} \\
& =S_{a}^{\top}\left(\mathcal{D}+J^{-1} T\right)
\end{aligned}
$$

where $T$ is the control torque and $\mathcal{D}$ is defined as:

$$
\mathcal{D}=\frac{1}{2} P\left[\left(\bar{q}_{e} \times\right\rangle \omega_{e}+q_{e 4} \bar{q}_{e}\right]+J^{-1}\langle\omega \times\rangle J \omega-\dot{\omega}_{d}
$$

To obtain the system stability, the input $T$ generated by the ACT can now be chosen as:

$$
T=-W_{a} J \operatorname{sgn}{ }^{e}\left(S_{a}\right)
$$

where $W_{a}>0$ is a design parameter, and $\operatorname{sgn}^{e}(\cdot)$ is an operator defined as $\operatorname{sgn}^{e}(V)=\left[\begin{array}{c}\operatorname{sgn}\left(v_{x}\right) \\ \operatorname{sgn}\left(v_{y}\right) \\ \operatorname{sgn}\left(v_{z}\right)\end{array}\right]$ for all $V=$ $\left[\begin{array}{lll}v_{z} & v_{y} & v_{z}\end{array}\right]^{\top} \in \mathcal{R}^{3}$. The above torque command can be shown valid if

$$
W_{a}>\|\mathcal{D}\|_{1}+\zeta, \quad \zeta>0 .
$$

Under such torque design, eq.(8) becomes:

$$
\begin{aligned}
\dot{V}_{a}\left(S_{a}\right) & =S_{a}^{\top}\left(\mathcal{D}+J^{-1} T\right) \\
& =S_{a}^{\top} \mathcal{D}-W_{a} S_{a}^{\top} s g n^{e}\left(S_{a}\right) \\
& \leq\left\|S_{a}\right\|_{1}\|\mathcal{D}\|_{1}-W_{a}\left\|S_{a}\right\|_{1} \\
& =\left\|S_{a}\right\|_{1}\left(\|\mathcal{D}\|_{1}-W_{a}\right) \\
& \leq-\zeta\left\|S_{a}\right\|_{1} \leq 0
\end{aligned}
$$

The above result implies that $S_{a}$ is bounded and will converge to zero in finite time.

Provided that $S_{a}=0$, the system dynamics are constrained by the following differential equations:

$$
\begin{aligned}
\dot{q}_{e} & =-\frac{1}{2}\left\langle\bar{q}_{e} \times\right\rangle P \bar{q}_{e}-\frac{1}{2} q_{e 4} P \bar{q}_{e} \\
\dot{q}_{e 4} & =\frac{1}{2} \bar{q}_{e}^{\top} P \bar{q}_{e}
\end{aligned}
$$

Define another Lyapunov function candidate $V_{e}\left(\bar{q}_{e}\right)=$ $\bar{q}_{e}^{\top} \bar{q}_{e}$, then

$$
\begin{aligned}
\dot{V}_{e}\left(q_{e}\right) & =2 \bar{q}_{e}^{\top} \dot{\bar{q}}_{e} \\
& =-\bar{q}_{e}^{\top}\left(\left\langle\bar{q}_{e} \times\right\rangle P \bar{q}_{e}+q_{e 4} P \bar{q}_{e}\right) \\
& =-q_{e 4} \bar{q}_{e}^{\top} P \bar{q}_{e}
\end{aligned}
$$


Since $q_{e 4}$ can be initially chosen a positive value in eq.(5) and is growing as can be seen in eq.(11), it is a positive all the time. From eq.(4),

$$
q_{1}^{2}+q_{2}^{2}+q_{3}^{2}+q_{4}^{2}=1
$$

which implies that $1 \geq q_{e 4}$, and hence $C \leq q_{e 4}(t) \leq$ $1, \forall t \geq 0$, so that the following relation will hold:

$$
-C \bar{q}_{e}^{\top} P \bar{q}_{e} \geq \dot{V}_{e}\left(\bar{q}_{e}\right) \geq-\bar{q}_{e}^{\top} P \bar{q}_{e}
$$

Hence $\bar{q}_{e}$ will converge to zero exponentially, and so will $\omega_{e}$ because $S_{a}=P \bar{q}_{e}+\omega_{e}=0$.

\subsection{Zero-Sliding Guidance Law}

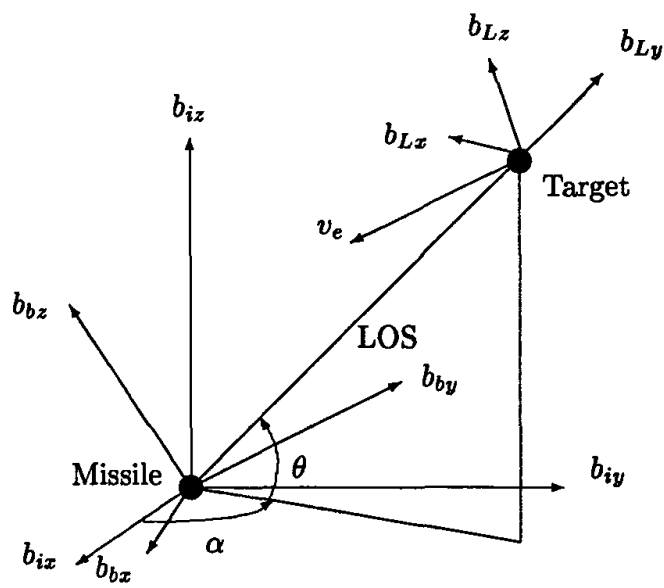

Figure 2: Missile Guidance Problem

In Fig.(2), $B_{i}=\left[\begin{array}{lll}b_{i x} & b_{i y} & b_{i z}\end{array}\right]$ is some inertial coordinate with its origin coincident with the gravity center of the missile, and $B_{b}=\left[\begin{array}{lll}b_{b x} & b_{b y} & b_{b z}\end{array}\right]$ is the missile body coordinate frame. Let $v_{e}$ be the relative velocity, whereas $\alpha$ and $\theta$ are the pitch and the yaw angles of the LOS (Line-of-Sight) with respect to the inertial coordinate $B_{i}$. We define a new LOS coordinate $B_{L}$ as follows: $b_{L y}$ is the unit vector along the LOS, $b_{L x}$ is the unit vector perpendicular to $b_{L_{y}}$ and parallel to the $x-y$ plane in inertial coordinate $B_{i}$ and $b_{L z}=b_{L x} \times b_{L y}$ is the third unit vector.

The principle of guidance law can be expressed in the following lemmas:

Lemma 1 Constant Bearing Condition: If the relative direction between the missile and the target can be kept fixed, than the missile will hit the target as long as the approaching velocity is positive and bounded away from zero.

Lemma 2 Zero-Sliding Guidance Law: Define the relative velocity $v_{e} \triangleq v_{t}-v_{m}$, where $v_{t}$ and $v_{m}$ are velocities of the target and the missile, respectively, and $R$ as the relative displacement vector from the missile to the target. If $v_{e}$ has no component on the normal direction of the $R$, and $v_{e}^{\top} R<0$, with $v_{e}$ being bounded away from zero, then the intercepting missile will eventually hit the target.

\section{Calculation of $v_{e}^{\perp}$ :}

Define sliding velocity $v_{e}^{\perp}$ as the normal components of $v_{e}$ with respect to $R$. From Lemma 2, the necessary condition of successful interception is the that $v_{e}^{\perp}=0$, and hence it will be suitable to choose $v_{e}^{\perp}$ as the control output of the guidance system.

From vector algebra, $v_{e}^{\perp}$ is equal to $v_{e}$ minus its projection on $R$, i.e.,

$$
v_{e}^{\perp}=v_{e}-\frac{R^{\top} v_{e}}{\|R\|^{2}} R
$$

and the time derivative of $v_{e}^{\perp}$ can be derived as:

$$
\dot{v}_{e}^{\perp}=\mathcal{A}\left(R, v_{e}\right)+\mathcal{B}(R) u
$$

where

$$
\begin{aligned}
\mathcal{A}\left(R, v_{e}\right) & =-\frac{\psi}{r^{2}} v_{e}^{\perp}+\frac{1}{r^{4}}\left(\psi^{2}-r^{2} v^{2}\right) R \\
\mathcal{B}(R) & =I_{3 \times 3}+\frac{1}{r^{2}}\left[\begin{array}{lll}
x_{e} R & y_{e} R & z_{e} R
\end{array}\right]
\end{aligned}
$$

and $v=\left\|v_{e}\right\|$ is the magnitude of the relative velocity, $u=\dot{v}_{e}$ is the acceleration to be treated as input at this stage, $\psi=R^{\top} v_{e}$ is the inner product of $v_{e}$ and $R, r=\|R\|$ is the distance between the missile and the target.

Assume that the magnitude of the acceleration generated by the DCT is $W_{t}$, then the pulsed control input can be expressed as

$$
u=-W_{t} \operatorname{sgn}^{v}\left(v_{\mathrm{e}}^{\perp}\right)
$$

where $W_{t}$ is the magnitude of the acceleration generated from the thrusters, and $s g n^{v}(\cdot)$ is an operator defined as

$$
\operatorname{sgn}^{v}(V)= \begin{cases}V & \text { if }\|V\| \neq 0 \\ \|V\| & \text { if }\|V\|=0 . \\ 0 & \text {. }\end{cases}
$$

for any $V \in \mathcal{R}^{3}$.

Under this controller design, eq.(14) becomes:

$$
\begin{aligned}
\dot{v}_{e}^{\perp} & =\mathcal{A}\left(R, v_{e}\right)+\mathcal{B}(R) u \\
& =-\frac{\psi}{r^{2}} v_{e}^{\perp}+\frac{1}{r^{4}}\left(\psi^{2}-r^{2}\left\|v_{e}\right\|^{2}\right) R-\mathcal{B} W_{t} \operatorname{sgn}^{v}\left(v_{e}^{\perp}\right) \\
& =-W_{t} \operatorname{sgn}^{v}\left(v_{e}^{\perp}\right)-\frac{\psi}{r^{2}} v_{e}^{\perp}+\frac{1}{r^{4}}\left(\psi^{2}-r^{2}\left\|v_{e}\right\|^{2}\right) R
\end{aligned}
$$

where we use the fact that $\mathcal{B} s n^{v}\left(v_{e}^{\perp}\right)=s g n^{v}\left(v_{e}^{\perp}\right)$ since $\left(v_{e}^{\perp}\right)^{\top} R=0$.

Define a Lyapunov function candidate as $V_{g}\left(v_{e}^{\perp}\right)=$ $\frac{1}{2}\left(v_{e}^{\perp}\right)^{\top} v_{e}^{\perp}$, then the time derivative of $V_{g}$ under control law eq.(16) can be evaluated as:

$$
\begin{aligned}
\dot{V}_{g}\left(v_{e}^{\perp}\right) & =\left(v_{e}^{\perp}\right)^{\top} \dot{v}_{e}^{\perp} \\
& =-\left(v_{e}^{\perp}\right)^{\top} W_{t} s g n^{v}\left(v_{e}^{\perp}\right)-\left(v_{e}^{\perp}\right)^{\top} \frac{\psi}{r^{2}} v_{e}^{\perp} \\
& \leq-\gamma_{1}\left\|v_{e}^{\perp}\right\| \leq 0
\end{aligned}
$$


if $W_{t}+\frac{\psi}{r^{2}}\left\|v_{e}^{\perp}\right\| \geq \gamma_{1}>0$ and $\left\|v_{e}^{\perp}\right\| \neq 0$. Hence, $v_{e}^{\perp}$ will be driven to zero in finite time as long as $\gamma_{1}$ is positive, i.e., provided that $W_{t}$ is larger than $\frac{\psi}{r^{2}}\left\|v_{e}^{\perp}\right\|$ by $\gamma_{1}$.

\subsection{Integration Analysis}

Assume that the missile can not exert acceleration on the axial direction, i.e., the DCT are arranged in the normal side along the missile body, the missile can only control the motion on the plane normal to the axial direction, which is called the active plane. If the attitude of the missile is aligned such that the commanded acceleration described by eq.(16) lies on the active plane of the missile, then the acceleration command can be modified as:

$$
\begin{aligned}
u= & u_{b x}+u_{b x} \\
= & -W_{t x} \operatorname{sgn}\left(b_{b x}^{\top} v_{e}^{\perp}\right) b_{b x} \\
& -W_{t z} \operatorname{sgn}\left(b_{b z}^{\top} v_{e}^{\perp}\right) b_{b z}
\end{aligned}
$$

The stability analysis of such design can simply follows the derivations as shown in eq.(18) and hence will be omitted here.

And to satisfy the attitude requirement, the desired attitude of the attitude controller is chosen as that of the LOS coordinate $B_{L}$ described in Section 3.2, and the angular velocity of the desired attitude is then defined as the necessary rotation of the LOS to eliminate $v_{e}^{\perp}$, and can be approximated by

$$
\omega_{d}=\frac{v_{e L z}}{r} b_{L x}-\frac{v_{e L x}}{r} b_{L z}
$$

To verify the stability of the overall system, we define the Lyapunov function candidate $V\left(S_{a}, S_{g}\right)$ of the overall system as:

$$
V\left(S_{a}, S_{g}\right)=\frac{1}{2} S_{a}^{\top} S_{a}+\epsilon S_{a}^{\top} S_{g}+\frac{1}{2} S_{g}^{\top} S_{g}
$$

where $S_{g}=v_{e}^{\perp}, S_{a}=P \bar{q}_{e}+\omega_{e}$, and $\epsilon$ is some positive constant. The torque command of the attitude controller is modified as:

$$
T=J W_{a} s g n^{e}\left(S_{a}+\mu S_{g}\right)
$$

where $\mu$ is some positive constant.

Note that

$$
\begin{aligned}
& -S_{a}^{\top} \operatorname{sgn}^{e}\left(S_{a}+\mu S_{g}\right) \\
= & -S_{a}^{\top} \operatorname{sgn}^{e}\left(S_{a}\right)-S_{a}^{\top}\left[\operatorname{sgn}^{e}\left(S_{a}+\mu S_{g}\right)-\operatorname{sgn}^{e}\left(S_{a}\right)\right] \\
\leq & -\left\|S_{a}\right\|+2 \mu\left\|S_{g}\right\|
\end{aligned}
$$

and similiarly

$$
-S_{g}^{\top} \operatorname{sgn}^{e}\left(S_{a}+\mu S_{g}\right) \leq-\left\|S_{g}\right\|+\frac{2}{\mu}\left\|S_{a}\right\|
$$

The time derivative of the Lyapunov function can be derived as:

$$
\dot{V}=\left(S_{a}+\epsilon S_{g}\right)^{\top}\left(\mathcal{D}+J^{-1} T\right)+\epsilon S_{a}^{\top} \dot{S}_{g}+S_{g}^{\top} \dot{S}_{g}
$$

$$
\begin{aligned}
\leq & S_{a}^{\top} \mathcal{D}+\epsilon S_{g}^{\top} \mathcal{D} \\
& -W_{a}\left(1-\frac{2 \epsilon}{\mu}\right)\left\|S_{a}\right\|-W_{a}(2 \mu-\epsilon)\left\|S_{g}\right\| \\
& +\epsilon S_{a}^{\top} \dot{S}_{g}-\lambda_{1}\left|c_{1}\right|-\lambda_{3}\left|c_{3}\right|-\frac{\psi}{r^{2}} c_{2}^{2}
\end{aligned}
$$

where $c_{1}=b_{b x}^{\top} v_{e}^{\perp}, c_{2}=b_{b y}^{\top} v_{e}^{\perp}, c_{3}=b_{b z}^{\top} v_{e}^{\perp}$, and $\lambda_{1}=$ $W_{t x}+\frac{\psi}{r^{2}}\left|c_{1}\right|, \quad \lambda_{3}=W_{t z}+\frac{\psi}{r^{2}}\left|c_{3}\right|$. Define $\sigma_{1}$ and $\sigma_{2}$ as:

$$
\begin{aligned}
\sigma_{1} & =W_{a}\left(1-\frac{2 \epsilon}{\mu}\right)-\left\|\mathcal{D}+\dot{S}_{g}\right\| \\
\sigma_{2} & =W_{a}(2 \mu-\epsilon)+\epsilon\|\mathcal{D}\|,
\end{aligned}
$$

then $\dot{V}\left(S_{a}, S_{g}\right)$ can be rewritten as:

$$
\begin{aligned}
\dot{V} \leq & \left\|S_{a}\right\|\left\|\mathcal{D}+\dot{S}_{g}\right\|+\epsilon\left\|S_{g}\right\|\|\mathcal{D}\| \\
& -\lambda_{1}\left|c_{1}\right|-\lambda_{3}\left|c_{3}\right|-\frac{\psi}{r^{2}} c_{2}^{2} \\
& -W_{a}\left(1-\frac{2 \epsilon}{\mu}\right)\left\|S_{a}\right\|-W_{a}(2 \mu-\epsilon)\left\|S_{g}\right\| \\
\leq & -\sigma_{1}\left\|S_{a}\right\|-\sigma_{2}\left\|S_{g}\right\|-\lambda_{1}\left|c_{1}\right|-\lambda_{3}\left|c_{3}\right|-\frac{\psi}{r^{2}} c_{2}^{2} \\
\leq & -\sigma_{1}\left\|S_{a}\right\|-\sigma_{2}\left(\left|c_{1}\right|+\left|c_{2}\right|+\left|c_{3}\right|\right) \\
& -\lambda_{1}\left|c_{1}\right|-\lambda_{3}\left|c_{3}\right|-\frac{\psi}{r^{2}} c_{2}^{2} \\
= & -\sigma_{1}\left\|S_{a}\right\|-\left(\sigma_{2}+\frac{\psi}{r^{2}}\left|c_{2}\right|\right)\left|c_{2}\right| \\
& -\left(\lambda_{1}+\sigma_{2}\right)\left|c_{1}\right|-\left(\lambda_{3}+\sigma_{2}\right)\left|c_{3}\right|
\end{aligned}
$$

where $\| S_{g}|| \leq\left|c_{1}\right|+\left|c_{2}\right|+\left|c_{3}\right|$ is employed in the above equation.

The conditions for stable system can be described in the following assumption:

Assumption 1 Through the entire course of guidance, the system satisfies

$$
\begin{aligned}
& \sigma_{1} \geq \varepsilon_{1}>0 \\
& \lambda_{1}+\sigma_{2} \geq \varepsilon_{2}>0 \\
& \lambda_{3}+\sigma_{2} \geq \varepsilon_{3}>0 \\
& \sigma_{2}+\frac{\psi}{r^{2}}\left|c_{2}\right| \geq \varepsilon_{4}>0
\end{aligned}
$$

where $\varepsilon_{i, i=1,2,3,4}$ are some positive constants.

By Lyapunov stability theorem, the system can be proved as an exponentially stable system. The sufficient conditions for successful interception can be summarized in the following theorem:

Theorem 1 If Assumptions 1 is satisfied, then the terminal guidance of the missile equipped with pulse type inputs is stable if the controller is designed as

$$
\begin{aligned}
u & =-W_{t x} \operatorname{sgn}\left(b_{b x}^{\top} v_{e}^{\perp}\right) b_{b x}-W_{t z} \operatorname{sgn}\left(b_{b z}^{\top} v_{e}^{\perp}\right) b_{b z} \\
T & =-W_{a} J \operatorname{sgn}{ }^{e}\left(S_{a}+\epsilon S_{g}\right)
\end{aligned}
$$

where $\epsilon$ is a small positive constant, and the desired attitude of the missile is chosen to that of the LOS coordinate. 


\section{Simulation}

Assume the target missile is initially at a distance of about $10 \mathrm{~km}$ and is flying with speed about $8 \mathrm{Mach}$, and the missile is initially set to speed $2.5 \mathrm{Mach}$, the total intercepting time is about 3 second. When the missile begin to intercept the target, it is assumed to have $10^{\circ}$ error in its attitude with the initial angular velocity of the missile set to zero.

The DCT continue to exert acceleration until about 3 second to eliminate the rotation of the LOS, whereas the ACT generate little torque command because the error quaternion is under the designed threshold of 0.1 to avoid the energy assumption resulting from the excessive torque output.

\begin{tabular}{|l||r|r|r|}
\hline Target & $\mathrm{X}$ & $\mathrm{Y}$ & $\mathrm{Z}$ \\
\hline \hline Position $(m)$ & 1830.13 & 1830.13 & 9659.26 \\
\hline Velocity $(m / s)$ & -604.913 & -507.582 & -2490.83 \\
\hline \hline Missile & $\mathrm{X}$ & $\mathrm{Y}$ & $\mathrm{Z}$ \\
\hline \hline Position $(m)$ & 0 & 0 & 0 \\
\hline Velocity $(m / s)$ & 129.242 & 108.447 & 730.778 \\
\hline
\end{tabular}

\section{Conclusions}

To intercept a target missile with pulse type input devices, a new guidance law is proposed, namely zerosliding guidance law, which aims at eliminating the rotation of the LOS between the missile and the target, and is formulated in $3 \mathrm{~d}$ space. The quaternion-based attitude controller is designed which guarantees exponentially tracking ability. Nonlinear system stability analysis and sliding mode control techniques are employed to fulfill the pulse type input, and the validity of the integrated system is also investigated. The feasibility of the design is verified through simulations.

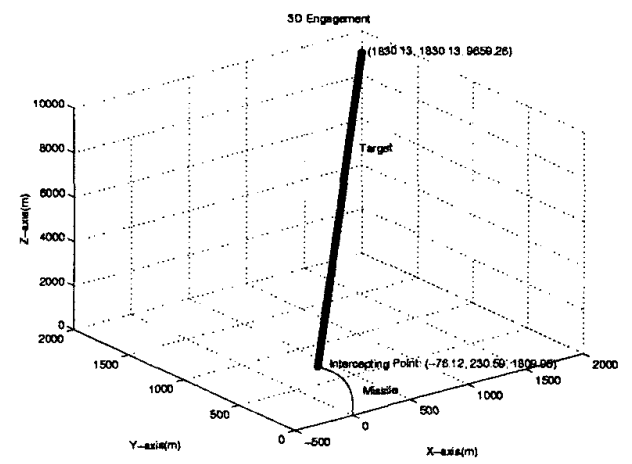

Figure 3: The 3D Engagement
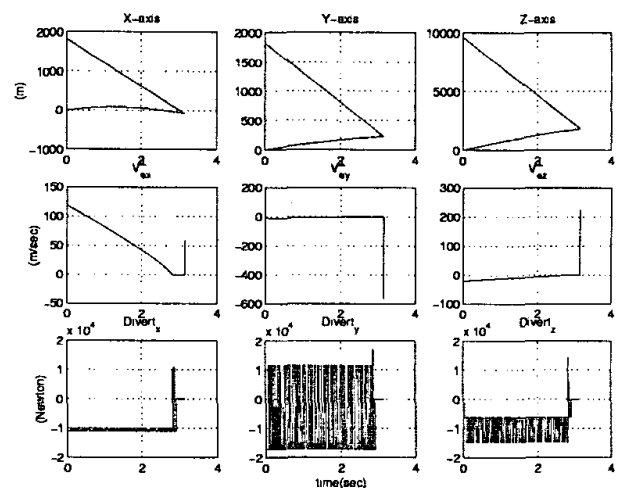

Figure 4: The Output Divert Forces
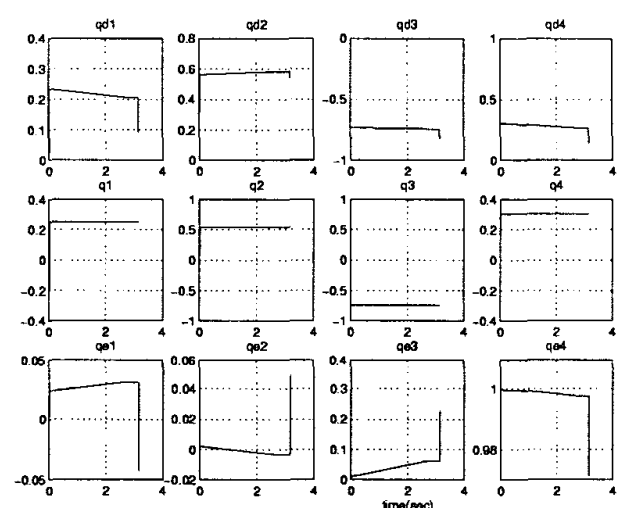

Figure 5: The Quaternion
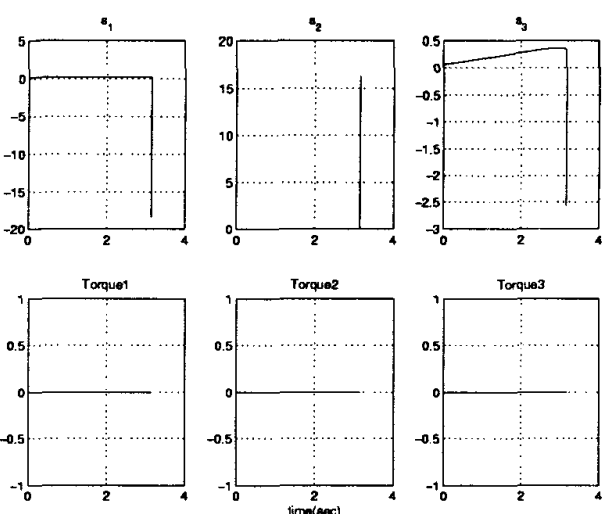

Figure 6: The Output Torque 